\title{
Prescribing Patterns of Surgical Oncologists: Are We Surgeons, Oncologists, or Both?
}

\author{
Results of a Society of Surgical Oncology Survey
}

\author{
Charles M. Balch, MD, FACS \\ Johns Hopkins Medical Institution, 600 N. Wolfe Street, Osler 624, Baltimore, Maryland
}

Although surgical treatment is the centerpiece of our specialty, what differentiates surgical oncology from other areas in surgery is the oncology experience and expertise needed in dealing with all aspects of cancer management in a multidisciplinary fashion. ... The salient feature of differentiation is that surgical oncology is both a technical and cognitive specialty involving a chronic disease process.

What is a surgical oncologist? Ann Surg Oncol 1993; 1:4

Despite our stated purpose of surgical oncology as a specialty, it would probably not surprise the readers of the Annals that there is still a fairly widespread perception among medical oncologists and members of the pharmaceutical companies that "surgeons spend their time in the operating room and have a relatively minor role in prescribing oncology and supportive drugs." Recently, I talked with a senior marketing executive of a major pharmaceutical company who stated that their company would not advertise in our journal, exhibit at our meeting, or fund SSO educational grants or sponsorships. This was because of their perception that surgeons - including surgical oncologists - neither prescribe their drug products nor influence their use in surgical patients. Perhaps less well understood by both the medical oncology community and the

Received July 27, 2007; accepted July 30, 2007; published online: September 5, 2007.

Address correspondence and reprint requests to: Charles $\mathrm{M}$. Balch, MD, FACS; E-mail: balchch@hjmi.edu

Published by Springer Science+Business Media, LLC $\odot 2007$ The Society of Surgical Oncology, Inc.
TABLE 1. On average, how many drug orders (inpatient or outpatient) do you or a trainee under your supervision prescribe each week? (520 responses)

\begin{tabular}{lcc}
\hline & Response percent & Response count \\
\hline 5 or less & $10.0 \%$ & 52 \\
$6-10$ & $15.2 \%$ & 79 \\
$11-15$ & $13.1 \%$ & 68 \\
$16-20$ & $9.8 \%$ & 51 \\
$21-25$ & $11.4 \%$ & 59 \\
More than 25 & $35.8 \%$ & 186 \\
None & $4.8 \%$ & 25 \\
\hline
\end{tabular}

pharmaceutical leadership is that many, if not the majority, of cancer patients look to the surgeon who made the diagnosis and initiated the primary treatment for advice about systemic therapy. Furthermore, surgical oncologists have led the way in defining predictors of survival outcomes for the majority of cancers, thereby identifying those at greatest risk of occult distant metastases, and hence are knowledgeable as to which of their surgical patients might benefit from systemic therapy.

While we may know this perception is untrue at an individual level, there is nothing better than having good data to break such molds of misperception. That is why I was pleased with the enormous response from our SSO members to a survey conducted by the Annals of Surgical Oncology Editors and Springer Verlag Publishers. One component of this survey was to tabulate prescribing practice patterns of 532 surgical oncologists. This is more than onethird of the active membership and makes the survey results very creditable and representative. (The entire 
TABLE 2. On average, how many times each week do you prescribe, recommend, or administer products in the following therapeutic categories?

\begin{tabular}{|c|c|c|c|c|c|c|}
\hline & $1-5$ & $6-10$ & $11-20$ & $>20$ & Never & Response count \\
\hline Analgesics & $14.7 \%$ & $32.2 \%$ & $25.5 \%$ & $24.2 \%$ & $3.4 \%$ & 475 \\
\hline Antibiotics, antivirals, antifungals & $35.3 \%$ & $32.3 \%$ & $16.5 \%$ & $11.8 \%$ & $4.3 \%$ & 468 \\
\hline Anticoagulation & $51.1 \%$ & $18.2 \%$ & $8.0 \%$ & $4.5 \%$ & $18.2 \%$ & 401 \\
\hline Anti-inflammatory agents (including steroids) & $49.9 \%$ & $23.1 \%$ & $9.9 \%$ & $5.5 \%$ & $11.7 \%$ & 403 \\
\hline Cardiovascular agents & $41.3 \%$ & $16.1 \%$ & $6.3 \%$ & $1.6 \%$ & $34.7 \%$ & 380 \\
\hline Diuretics & $48.1 \%$ & $18.1 \%$ & $2.1 \%$ & $2.3 \%$ & $29.5 \%$ & 387 \\
\hline Gastrointestinal agents & $36.7 \%$ & $25.4 \%$ & $9.8 \%$ & $4.8 \%$ & $23.4 \%$ & 398 \\
\hline Hormone agents & $43.5 \%$ & $14.1 \%$ & $6.0 \%$ & $2.8 \%$ & $33.7 \%$ & 398 \\
\hline Chemotherapeutic agents & $20.9 \%$ & $4.8 \%$ & $3.2 \%$ & $1.3 \%$ & $69.8 \%$ & 374 \\
\hline Growth factors (bone marrow) & $24.9 \%$ & $3.0 \%$ & $0.3 \%$ & $0.3 \%$ & $71.6 \%$ & 366 \\
\hline Heparin products/antithrombosis & $35.9 \%$ & $22.1 \%$ & $12.3 \%$ & $10.0 \%$ & $19.7 \%$ & 390 \\
\hline Monoclonal antibodies & $16.0 \%$ & $5.2 \%$ & $0.0 \%$ & $0.0 \%$ & $78.8 \%$ & 368 \\
\hline Neuropsychiatric agents & $39.1 \%$ & $5.5 \%$ & $1.1 \%$ & $0.3 \%$ & $54.1 \%$ & 366 \\
\hline Respiratory agents & $44.0 \%$ & $11.4 \%$ & $4.7 \%$ & $1.9 \%$ & $38.0 \%$ & 361 \\
\hline
\end{tabular}

survey results will be sent to SSO members in a separate mailing and can be viewed online at its website http://www.surgonc.org/.)

Of the 532 surgeons who responded, almost $71 \%$ listed their workplace as a university/teaching hospital, and $16 \%$ are in private practice (the remainder are in other categories). Their surgical oncology specialty interests were gastrointestinal (55\%), breast $(54 \%)$, melanoma (31\%), hepatic/pancreatic (28\%), endocrine $(12 \%)$, and sarcomas (12\%) (up to three areas could be identified). They were busy clinicians, as illustrated by the fact that, on average each week, $45 \%$ of respondents saw $26-50$ patients, $18 \%$ saw $51-$ 75 patients, and $8 \%$ saw more than 75 patients. More than $83 \%$ referred their surgical patients to a prescribing medical oncologist "often" or "very often."

Surgical oncologists do indeed prescribe drugs frequently ... perhaps at a rate that would surprise some who are outside our field. When asked: "on average, how many drug orders do you or a trainee under your supervision prescribe each week," more than one-third prescribed 25 or more drugs and an additional one-third prescribed 11-25 drugs (see Table 1). The breakdown of therapeutic drug categories confirms what would be expected for a high frequency of prescribing drugs as a component of perioperative management (analgesics, antibiotics, antiemetics). In addition, two-thirds of surgical oncologists each week either prescribed, recommended, or administered oncology-related hormone agents. In addition, $30 \%$ did so for chemotherapeutic agents and growth factors and $21 \%$ did so for monoclonal antibodies (Table 2). Finally, when asked "Over the last twelve months, have you ordered or prescribed a recently approved therapy or a therapy under investigation?" $51 \%$ responded affirmatively.

The results of this survey, the largest on this subject ever published, clearly demonstrate that our membership embraces a dual role of being surgeon and oncologist. The management of the surgical patient with cancer involves the blending of physician skills and experience that uses both their technical skills in the operating room and their cognitive skills in using drugs and other systemic agents to get patients safely through the perioperative period. These cognitive skills are also used to manage patients' cancer with systemic agents as a member of the multidisciplinary team.

The survey has some implications for the SSO and its membership. First, it should continue to promote the highest-quality multidisciplinary patient care and research through educational activities at its Annual Meeting and publications in the Annals of Surgical Oncology. Second, it should continue to emphasize and teach the indications, risks, and benefits of using various drugs and systemic agents in surgical training programs. Third, it should continue to incorporate the testing and validation of cancer drugs and perioperative drugs through clinical trials. Fourth, it should continue to actively solicit grants and sponsorships from the pharmaceutical and biotechnology sector to help support the SSO's educational, research, and training missions. With all these ongoing activities, the SSO and its membership will continue to bring highquality and comprehensive cancer care to our surgical patients as both surgeon and oncologist. 